\title{
Broschüre schult Fachpersonal
}

— Bei der Behandlung neuroendokriner Tumoren (NET) spielt die korrekte s.c.A- oder i.m.BInjektion von SSA (Somatostatin-Analoga) eine wesentliche Rolle. Da Ärzte diese an geschultes Fachpersonal delegieren können, hat Novartis Oncology zur Unterstützung des medizinischen Fachpersonal eine Broschüre herausgebracht: „Der NET-Patient: Sicherheitsaspekte für Pflege- und Fachpersonal bei der SSA-Applikation". Diese Broschüre erhalten auch die Teilnehmer der NET-Pflegeakademie, ein Fortbildungskonzept für Pflegepersonal von Novartis Oncology.

Die Broschüre enthält umfassende Informationen zur Behandlung mit SSA, erläutert aber auch Schritt für Schritt die korrekte s.c.- oder i.m.-Injektionstechnik. Ergänzt wird das durch einen Überblick zu den verfügbaren SSA und Informationen zur generellen Sicherheit der Applikationen. Sie beantwortet zudem Fragen wie „Welche Pulver-Durchfeuchtungszeit wird bei der Suspensionherstellung empfohlen?" oder "Wie stelle ich auch bei schlanken Patienten eine subkutane Injektion sicher?". Neben Tipps ausgewählter Experten, liefert sie Informationen zum Transport von SSA auf Reisen, zur Aufbewahrung und zu Neben- und Wechselwirkungen. Die Broschüre stellt auch den kostenlosen und flexiblen Patientenservice "SanService" von Novartis vor: Im Rahmen dieses Service kommt eine qualifizierte Pflegekraft zu dem NET-Patienten nach Hause und verabreicht dort das SSA.

www.leben-mit-net.de

\section{Inkontinenz auffangen}

\section{Extra dichte Stoffhöschen ersetzen Einlagen}

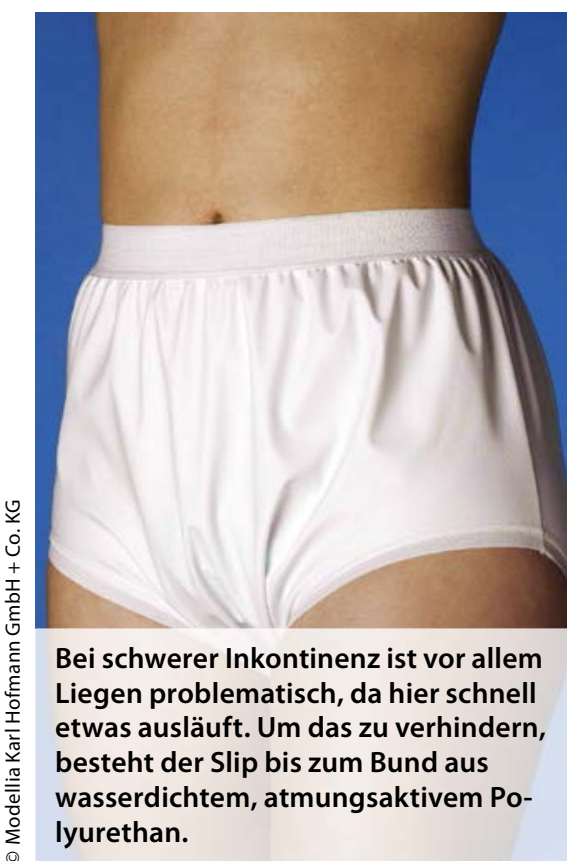

— Vom leichten Tröpfeln bis hin zum unbemerkten Überlaufen - Inkontinenz ist für die Betroffenen meist sehr beschämend. Für das Pflegepersonal oder pflegende Angehörige kann das zum Hygieneproblem werden, etwa wenn Windeln oder Einlagen vom Patienten abgelehnt werden. Auch vergessen vor allem Senioren mitunter, dass sie diese Hilfsmittel eigentlich bräuchten und ziehen herkömmliche Höschen an, die aber keine Schutzwirkung haben. Eine gewohnte und dennoch sichere Alternative bietet hier die eigens entwickelte Inkontinenz-Unterwäsche von Modellia: Die Stoffhöschen wirken wie normale Wäsche, allerdings fangen eingearbeitete Kunststoffmembranen auf, was daneben geht. Antimikrobielle Materialien verhindern zudem die sonst typischen Gerüche, und die spezielle Passform hält Einlagen sicher am Platz.

Für leichte Inkontinenz gibt es sogar Slips mit waschbarer Microfaser-Saugzone, die an der Oberfläche trocken bleiben und die Einlagen ersetzen. Je nach Schwere der Inkontinenz gibt es Varianten mit verschieden großen PU-Teilen bis hin zu einem Modell, das komplett aus dem auslaufsicheren Material besteht und vor allem für bettlägerige Patienten oder für nachts gedacht ist. Damit ist sichergestellt, dass auch im Liegen Nachtwäsche und Bett sauber bleiben.

www.modellia.de
Neues Konzept

\section{Für jede Indikation die richtige Matratze}

- Mit einem neuen Konzept wird das Matratzenmanagement für die unterschiedlichen Pflegeanforderungen der Bewohner in einer stationären Pflegeeinrichtung erheblich erleichtert. Es bietet die Möglichkeit, nicht nur für jede Indikation die richtige Matratze einzusetzen, sondern auch die Rückverfolgbarkeit des Einsatzes zu organisieren und nachzuhalten. Durch die unterschiedlichen Bezugsfarben (PU Qualität) der Matratze lässt sich rasch erkennen, ob es sich um eine Basic, Komfort oder Prophylaxe/Therapiematratze handelt. Die PU-Bezüge sind abnehmbar, waschbar bis $95 \mathrm{Grad}$, diffusionsfähig, texturiert, zertifiziert nach Öko-Tex Standard 100.

Für das WIBU-Matratzensortiment wurden die hochwertigen Metzeler Pflegematratzen gewählt, da sie mit neuen und bewährten SchaumTechnologien einen wichtigen Beitrag für die Pflegequalität und die unterschiedlichen Anforderungen leisten können. Ganz neu sind die Anti-Dekubitus-Matratzen MetzoCare I + II. Letztere - im speziellen druckentlastenden Dreischichtaufbau - wurde zur Dekubitus-Prophylaxe und -Therapie entwickelt und leistet ihren Beitrag bis zu hohem Dekubitusrisiko und Therapieunterstützung (Grad 1-4), wenn alle anderen Maßnahmen berücksichtigt werden. Die Matratze wurde der amtlichen Prüfung zur Druckentlastung durch das Institut der TU Berlin unterzogen und mit einer Bestnote (Höchstklasse H) zertifiziert.

Das WIBU-Matratzensortiment bietet aber auch Matratzen mit hohem Liegekomfort, die sich besonders zur Entspannung und Regeneration des Bewegungsapparates eigenen, sowie hochwertige Universal- und eine Schwerlastmatratze.

www.wibu-gruppe.de 